\title{
La filosofía como método de enseñanza y aprendizaje en la educación primaria
}

\section{The philosophy like a learning method in elementary school}

\author{
Gabriela Alejandra Contreras Muñiz ${ }^{1}$ \\ Laura Cristina Baca Calderón² \\ Adela Cardona Hernández ${ }^{3}$
}

\begin{abstract}
Resumen
Existen dos aspectos del Plan y Programa de Estudios de la educación básica que responden a la formación de un individuo crítico y reflexivo, el medio por el cual el docente puede cumplir estos objetivos es la filosofía, ciencia que no solo debe estar destinada a la educación media o superior, sino que por su naturaleza puede utilizarse como un método de aprendizaje y catalizador de este mismo, además de obligar al alumno a cuestionarse constantemente dicho aprendizaje como una forma de convertir un espacio de cuatro paredes en una verdadera aula de investigación.
\end{abstract}

\section{Palabras clave}

Crítico, reflexivo, formación, filosofía, aprendizaje.

\begin{abstract}
${ }^{1}$ Gabriela Alejandra Contreras Muñiz. Institución Benemérita y Centenaria Escuela Normal del Estado de Chihuahua Profesor Luis Urías Belderráin, México. Licenciatura en Educación Primaria. Correo electrónico: contrerasgabriela294@yahoo.com.mx

ID: http://orcid.org/0000-0003-4776-7711
\end{abstract}

${ }^{2}$ Laura Cristina Baca Calderón. Institución Benemérita y Centenaria Escuela Normal del Estado de Chihuahua Profesor Luis Urías Belderráin, México. Licenciatura en Educación Primaria. Correo electrónico: bacacalderonlaura@gmail.com ID: http://orcid.org/0000-0002-8159-2591

3 Adela Cardona Hernández. Docente de la Institución Benemérita y Centenaria Escuela Normal del Estado de Chihuahua Profesor Luis Urías Belderráin, México. Ha participado en varios congresos de Investigación Educativa, nacionales e internacionales. Correo electrónico: a.cardona@ibycenech.edu.mx

ID: http://orcid.org/0000-0001-8347-1470 
RECIE. Revista Electrónica Científica de Investigación Educativa Vol. 4, núm. 2, enero-diciembre 2019, pp. 867-873.

\begin{abstract}
There are two aspects of the Basic Education Curricula that respond to the formation of a critical and reflective individual, the means by which the teacher can fulfill these objectives is philosophy, a science that should be destined not only to higher education, but because of its nature it can be used as a method and catalyst of learning, forcing the student to constantly question this learning and also as a way of turning a four-wall space into a real research classroom.
\end{abstract}

\title{
Keywords
}

Critical, reflexive, formation, philosophy, learning.

\section{Introducción}

Puede parecer en un principio por el título, que se trata de una idea extraña o fuera de lugar, pero en el presente documento se trata de plantear esta posibilidad, no de enseñar filosofía, sino de filosofar con niños, y que mejor edad para comenzar con la tarea del pensar reflexivo que desde la niñez, que se caracteriza por una capacidad de asombro y curiosidad innata. Durante nuestras prácticas hemos podido constatar la poca capacidad de reflexión y critica de los alumnos lo anterior posiblemente tiene su causa en la carencia de actividades y estrategias que favorezcan a estos aspectos, las numerosas tareas que tiene que realizar el docente día con día agotan casi la mayor parte de su tiempo, el cual aprovecha en asegurar más la memorización de los contenidos por parte de los alumnos, que una verdadera critica de los mismos, esto es comprensible se supone que el estudiante debe cumplir con varios aprendizajes esperados y competencias en el transcurso del año escolar y en el examen no se le cuestionará abiertamente su perspectiva de la revolución mexicana o que es el amor, la justicia o la libertad, a no ser que la respuesta sea escogida casi de forma robótica de las opciones múltiples presentadas en el examen.

La capacidad de pensar no es algo que simplemente se da por hecho, sino que realmente es una tarea difícil si es a profundidad, durante la Convención de los Derechos del Niño celebrada en 1989 garantiza entre otros el derecho de expresar su opinión libremente (artículo 12) la libertad de expresión (artículo 13) y la libertad de pensamiento (artículo 14).

La filosofía permite al docente transitar de un área de conocimiento a otra, así como relacionarlas sin importar cuales sean, mediante métodos como el dialogo, el debate, se trata de implementar una pedagogía de la pregunta y no de la respuesta en la que el papel del docente sea solo un 
modulador o guía para el alumno descubra, experimente y actué frente a la realidad, también y hace hincapié en la educación para la paz, así como en la formación cívica y ética para la formación de una sociedad más justa y democrática.

A pesar de que el tema no es nuevo, se considera que su vigencia no tiene límite y que bien vale la pena poner en práctica en las aulas de un país con grandes problemas de participación social como lo es el nuestro.

\section{Desarrollo}

Matthew Lipman es uno de los principales teóricos en torno a este tema, en su libro "El lugar del pensamiento en la educación" menciona la importancia de cultivar tres dimensiones, la crítica, la creativa y la cuidadosa, plantea la idea de fomentar en el alumno el desarrollo multidimensional al contrario de la educación desde un punto de vista más empirista, el autor que propone como meta el equilibrio reflexivo mediante el cual el niño permanecerá en la constante revisión, modificación e investigación del conocimiento, sin conformarse con una única verdad.

Para Lipman el pensamiento se comporta y rige bajo diversos valores o criterios, el pensamiento puede ser analítico, lógico, creativo, intuitivo, imaginativo etc. "Unas veces, el pensamiento se mueve suave y rutinariamente como los vagones de un tren en sus raíles; otras, campa libremente como un pájaro" (Lipman, 2016, p. 16). En las diversas experiencias de prácticas se ha observardo que el docente implementa estrategias muy parecidas en cuanto al tipo de pensamiento que se necesita, a pesar de ser materias diferentes; no puede olvidar que mientras se imparte una clase, miles de pensamientos atraviesan por la mente de los niños y no necesariamente relacionados con el tema así mismo se debe considerar que si se trata de una asignatura como matemáticas, el tipo de pensamiento predominante será lógico, este no puede aplicarse de la misma forma en materias como historia o las artes. Una constante en nuestra práctica docente es la enorme necesidad del alumno de ser escuchado, no como un alumno más sino como un individuo con pensamientos, sentimientos y emociones propias que ve en la escuela la oportunidad de participar significativamente en algo, ya sea un proyecto, un trabajo por sencillo que sea o conversación; de nuevo tanto padres de familia como el propio maestro impiden su expresión.

Cuando la educación era por completo de transmisión, poco importaban los procesos cognitivos, pero conforme la sociedad y la ciencia avanzaban se vio la necesidad de incluir estos aspectos que tenían como máximo representante al psicopedagogo Jean Piaget, en su teoría de los estadios se encuentra la etapa de operaciones concretas en la cual el niño 
puede resolver diversos problemas que se le plantean y aunque Piaget lo ejemplifica en el área de las matemáticas, tanto el Plan y Programas 2011 como el nuevo Modelo Educativo que aún no entra en vigor recalca la importancia de que el alumno sea capaz de enfrentarse a problemas que asemejen situaciones reales, en este sentido el alumno que posea la capacidad de pensar de forma multidimensional será más competente al resolver dichas problemáticas. En el pasado durante las jornadas de prácticas aplicaron estrategias de matemáticas en un grupo de sexto grado, que consistieron en escribir un cuento que tuviera como desenlace la resolución de un problema de fracciones, no sin antes mostrar un ejemplo de un cuento matemático narrado en un video, cuál fue la sorpresa que solo tres alumnos de veintinueve realizaron la encomienda, el resto se limitó a formular un problema, hecho que hizo reflexionar sobre la poca o nula importancia que se tiene en las aulas por fomentar el pensamiento creativo, como si no fuera parte fundamental de la educación básica y del perfil de egreso. Es necesario realizar más actividades como la anterior para retar al niño a utilizar más de una inteligencia. Lipman (2016) afirma:

El pensamiento multidimensional, tal como lo entendemos aquí, apunta a un equilibrio entre lo que es cognitivo y lo que es afectivo, entre lo perceptivo y lo conceptual; entre lo físico y lo mental, entre lo que es gobernado por reglas y lo que no (p. 18).

El segundo autor al que se hace mención es Oscar Brenifier, con la intención de estudiar algunos aspectos de su obra "La práctica de la filosofía en la escuela primaria" como inicio el autor se cuestiona sobre la tardía labor de la educación filosófica, el alumno de preparatoria se tiene que enfrentar con el enorme reto que le impone la actividad reflexiva en la asignatura de filosofía, lamentablemente no es la única materia a la que los alumnos relegan o desprecian con la justificación de que carece de importancia además de ser aburrida, se ha advertido en los estudiantes una profunda apatía por la investigación y la producción de conceptos, pareciera que la tecnología más que ayudar académicamente tanto a niños como jóvenes está representando una barrera de aprendizaje, porque ya está todo al alcance de un clic, la lectura no se analiza ni mucho menos se critica, no se hace nada productivo de ella, muestra de ello se vive en las practica profesionales, el trabajo consiste en elaborar un relato histórico a partir de la investigación en diversas fuentes, principalmente internet, sorprendentemente los estudiantes desarrollaron si mucho cinco renglones de su tema, aquellos que llevaron una investigación más extensa fue porque imprimieron la información, sin embargo esta no concordaba con el tema del trabajo por lo 
que deduje que ni siquiera se habían dado a la tarea de leer, simplemente copiaron y pegaron lo primero que encontraron.

Otra característica que se acentúa en las prácticas se relaciona con la subestimación de las capacidades de los niños, tal vez al presentar éste trabajo de investigación el docente pueda objetar que el alumno no tiene la capacidad para apropiarse del conocimiento por medio de la filosofía, pero no se pretende que se convierta en un genio o que haga grandes descubrimientos cuando lo más importante es descubrirse a sí mismo. Como Sócrates se dará la libertad al infante de reflexionar cualquier cosa que se presente en su mundo, esto no significa darle rienda suelta a cualquiera de sus deseos e impulsos, en este sentido Brenifier plantea tres dimensiones: Dimensión intelectual: pensar por uno mismo, que abarcan los siguientes aspectos: proponer conceptos e hipótesis, comprender sus ideas, analizar, reformular etc., la dimensión existencial: ser uno mismo: expresar su identidad personal por medio de sus juicios y sus elecciones y asumirla. Y la dimensión social que tiene que ver con las relaciones sociales, los valores y la sana convivencia. Durante un día normal de clases e pueden presentar múltiples disputas entre los alumnos que tienen origen por cosas tan simples como la negación a prestar un lápiz, hasta ofensas mayores que pueden dañar la autoestima de los niños, para esto el docente tiene que hacer de juez y la mayoría de las veces termina por imponer un castigo a todos los involucrados ya que le es imposible saber exactamente cómo sucedieron los hechos, la filosofía puede ser el medio por el cual el niño logre comprender por sí mismo la importancia del respeto, el valor de la justicia, así como la repercusión de sus actos.

Para esto el autor tiene varias estrategias para trabajar en el aula, una de ellas se titula ¿qué hay de nuevo? Y que tiene como uno objetivo la charla abierta por parte de cada uno de los alumnos acerca de sus vidas y lo que sucede en ellas, ciertamente uno de los problemas que más se ve actualmente es la falta de atención de los niños por parte de sus padres, es entonces que la escuela resulta el único lugar en el que se sienten libres para hablar de sí mismos, sus motivaciones, gustos, preocupaciones etc. Sin embargo, incluso el maestro les niega esta posibilidad, esto no tiene nada que ver con motivos personales, simplemente el docente se ve abrumado ante las múltiples tareas que a su profesión se le asignan, pero esto no debe ser un problema siempre y cuando los o los docentes sepan adaptar sus clases y sean flexibles ante nuevas posibilidades. 
RECIE. Revista Electrónica Científica de Investigación Educativa Vol. 4, núm. 2, enero-diciembre 2019, pp. 867-873.

\section{Preguntas de Investigación}

- ¿Cómo vincular la filosofía para niños en los contenidos curriculares?

- ¿Qué factores benefician el desarrollo del Pensamiento críticocreativo en la educación y en la sociedad?

\section{Metodología}

La metodología empleada en el trabajo de investigación, pretende dar a conocer la concepción de los diversos actores de la indagación que se realizó con veintiséis alumnos de quinto grado, que su edad fluctúa entre los diez y once años de la escuela Independencia en la ciudad de Chihuahua, Chih. La presente investigación parte del enfoque cualitativo con un diseño fenomenológico pues "se enfocan en las experiencias individuales subjetivas de los participantes...se pretende reconocer las percepciones de las personas y el significado (para ellas) de un fenómeno u experiencia" (Hernández, Fernández, \& Baptista, 2010)(Hernández, Fernández y Baptista 2006, p.712). Se emplearon técnicas como la observación y la entrevista así como el grupo focal para la recopilación de datos.

Los sujetos que participaron en esta investigación pertenecen a una clase media baja en un contexto urbano al norte de la ciudad, durante el proceso de la jornada de práctica intensiva que se observó en base a una guía previamente diseñada y piloteada, y bajo categorías propias de la práctica profesional además de enfocando algunos indicadores en la identidad docente.

\section{Conclusiones}

La filosofía como método de enseñanza y aprendizaje en la educación primaria es todo un reto ya que se necesitan crear las estrategas necesarias para llevar al alumno a desarrollar su pensamiento reflexivo y analítico para que conozca sus habilidades y destrezas las cuales serán de ayuda para su pensamiento analítico el cual será utilizado a lo largo de su vida, considerando que la infancia es una etapa fundamental para incentivar a los niños a reflexionar para ello se necesita del apoyo de su contexto externo y contexto escolar.

Se dice que la filosofía es una asignatura más, pero la realidad es que tiene un amplio desarrollo que refuerza el conocimiento de los seres humanos, en primera instancia es parte del desarrollo del pensamiento, y no necesariamente se necesita un tema de aprendizaje si no que para cualquier situación se puede implementar el pensamiento reflexivo, a lo largo de la vida 
se presentan situaciones, en las cuales se tendrá que reflexionar con profundidad para llegar un resultado o a una decisión.

$\mathrm{Al}$ ser el aula el primer escenario en donde los alumnos tienen que aprender a ser independientes, a tomar decisiones por sí mismos y desarrollar habilidades cognitivas, el docente juega un papel fundamental para impulsar a el alumno a lograr todas sus metas creando un lugar en donde puedan sentirse seguros, de este modo se lograra que el alumno se sienta en confianza y pueda aprender a resolver problemas que impliquen a los diferentes aprendizajes pero del mismo modo pueda resolver problemas que lo involucren con su entono, ya sean familiares, sociales o institucionales.

La filosofía es una ciencia de una complejidad hermosa y por medio de ella el niño puede madurar tanto intelectual, emocional y personal siendo posible que acceda a diversas áreas del conocimiento sin sentirse atado de ninguna forma, es decir una persona libre y la libertad probablemente junto con la felicidad son algunos de los más importantes valores a los que aspira cualquier educación que se presuma actual y con fundamentos humanísticos, en este sentido es la filosofía la ciencia más completa y perfecta mediante la cual el alumno pueda construir su propio camino.

\section{Referencias}

Brenifier, O. (2012). La práctica de la filosofía en la escuela primaria. Valencia Diálogo

Joanna Haynes: Los niños como filósofos. El aprendizaje mediante la indagación y el dialogo en la escuela primaria, Ed. Paidos educador, Barcelona, 2002.

Lipman, M. (2016). El lugar del pensamiento en la educación. Barcelona: Octaedro.

VELASCO, C. F. (2017). Proyecto de investigación filosofia para niños: un reto para la educacion ètica $\mathrm{y}$ en valores. Obtenido de http://repository.usta.edu.co/bitstream/handle/11634/9328/Velas coFernando2017.pdf?sequence=1\&isAllowed =y

Félix García-Moriyón, R. C. (2000). Valoraciòn de «Filosofía para Niños»:. Redalyc, 568-571.

http://repository.usta.edu.co/bitstream/handle/11634/9328/VelascoFern ando2017.pdf?sequence $=1$ \&isAllowed $=y$

http://elbuho.aafi.es/buho11/fihttps://www.rafaelrobles.com/2012/11/la -practica-de-la-filosofia-en-la-escuela-primaria-2/losofianinos.pdf 
RECIE. Revista Electrónica Científica de Investigación Educativa Vol. 4, núm. 2, enero-diciembre 2019, pp. 867-873. 